\title{
Pseudomonas aeruginosa: Study of Antibiotic Resistance and Molecular Typing in Hospital Infection Cases in a Neonatal Intensive Care Unit from Rio de Janeiro City, Brazil
}

\author{
MM Loureiro, BA de Moraes, VLF Mendonça, MRR Quadra*, GS Pinheiro*, MD Asensi/+
}

Laboratório de Enterobactérias, Departamento de Bacteriologia, Instituto Oswaldo Cruz-Fiocruz, Av. Brasil 4365, 21045-900 Rio de Janeiro, RJ, Brasil *Hospital Maternidade Alexander Fleming II, Sistema Único de Saúde, Rio de Janeiro, RJ, Brasil

This study had the objective of to analyze the demographic and bacteriologic data of 32 hospitalized newborns in an neonatal intensive care unit of a public maternity hospital in Rio de Janeiro city, Brazil, seized by Pseudomonas aeruginosa sepsis during a period ranged from July 1997 to July 1999, and to determine the antimicrobial resistance percentage, serotypes and pulsed field gel electrophoresis (PFGE) patterns of 32 strains isolated during this period. The study group presented mean age of 12.5 days, with higher prevalence of hospital infection in males (59.4\%) and vaginal delivery (81.2\%), than females (40.6\%) and cesarean delivery (18.8\%), respectively. In this group, $20(62.5 \%)$ patients received antimicrobials before positive blood cultures presentation. A total of $87.5 \%$ of the patients were premature, $62.5 \%$ presented very low birth weight and $40.6 \%$ had asphyxia. We detected high antimicrobial resistance percentage to $\beta$-lactams, chloramphenicol, trimethoprim/sulfamethoxazole and tetracycline among the isolated strains. All isolated strains were classified as multi-drug resistant. Most strains presented serotype O11 while PFGE analysis revealed seven distinct clones with isolation predominance of a single clone (75\%) isolated from July 1997 to June 1998.

Key words: Pseudomonas aeruginosa - neonatal intensive care unit - hospital infection - sepsis - Rio de Janeiro - Brazil

Pseudomonas aeruginosa is one of the most common pathogens involved in hospital infection (HI) causing opportunistic infections in humans, particularly among immunocompromised patients (Bert \& Lambert-Zechovsky 1996, Kinoshita et al. 1997, Tsakris et al. 2000), and because of its ubiquitous nature, ability to survive in adverse conditions, and affinity for moist environments, remains a common pathogen in intensive care units (ICU) (Grundmann et al. 1995, Moolenaar et al. 2000)

The HI causes complication of medical care in ICUs, principally in neonatal intensive care units (NICU), due to the natural immunodeficiency of newborns, submission to invasive procedures of therapy and diagnostic, presence of several underlying risk conditions and indiscriminated antimicrobial use, and can causes significant morbidity and mortality (Sader et al. 1993, Kettner et al. 1995, Moolenaar et al. 2000). Mortality upon infected patients with $P$. aeruginosa sepsis was $46.7 \%$, compared to only $21 \%$ for other bacteria in a study that collected blood stream isolates from nearly 50 medical center in the USA (Jones et al. 1997).

The worldwide emergence of multi-resistant bacterial strains is a growing concern, especially in $\mathrm{HI}$ cases caused by $P$. aeruginosa. Among nosocomial bacterial infections, those caused by $P$. aeruginosa are associated with highest mortality rate, and are difficult to eradicate from in-

Financial suppor: $\mathrm{CNPq}$ and Papes (Fiocruz)

${ }^{+}$Corresponding author. Fax: +55-21-2270.6565. E-mail: marise@ioc.fiocruz.br.

Received 23 May 2001

Accepted 4 February 2002 fected tissues or blood because those microorganisms are virulent and have a limited susceptibility to antimicrobials (Kettner et al. 1995, Harris et al. 1999).

The epidemiology of $P$. aeruginosa infections are usually studied by the analysis of phenotypic markers, including biotype, serovar, pyocin production, phage type, and antimicrobial susceptibility pattern (Pitt 1988, Kinoshita et al. 1997). Typing of strains is important for eradication of environmental sources as well as prevention of cross-infections and monitoring of antimicrobial therapy efficacy (Poh et al. 1992). Recently, nucleic acidbased methods have been used to assist identification of bacteria to subspecies level in epidemiological studies, providing a higher discriminatory power than phenotypic parameters (Severino et al. 1999)

Chromosomal DNA restriction analysis by pulsed field gel electrophoresis (PFGE) is considered worldwide the most powerful tool to perform hospital epidemiologic studies of $P$. aeruginosa because of its high discriminatory capacity. These technique facilitate the elucidation of transmission routes, genetic variability and phylogenetic distances between strains (Sader et al. 1993, Grundmann et al. 1995, Renders et al. 1996, Müller-Premru \& Gubina 1999)

This study reports the demographics characteristics of hospitalized newborns seized by $P$. aeruginosa sepsis associated with $\mathrm{HI}$, and antimicrobial resistance percentage, serotypes and PFGE patterns of the isolated strains in a NICU from a public maternity hospital in Rio de Janeiro city, Brazil.

\section{MATERIALS AND METHODS}

Hospital and patients - Between July 1997 and July 1999, 32 P. aeruginosa strains were isolated from blood cultures of different NICU newborns involved in HI cases, 
at the Hospital Maternidade Alexander Fleming II (HMAF), Rio de Janeiro city, Brazil. It is a maternity hospital providing assistance and perinatal care including a neonatology intermediate care unity (NIU) with 40 beds and a NICU with 15 beds.

HI cases were defined according to Centers for Diseases Control and Prevention (CDC) (Garner et al. 1988). In general, infections that occurred after $48 \mathrm{~h}$ of permanence at the hospital were assumed to be hospital acquired.

Blood cultures, strains identification and susceptibility testing - $0.2 \mathrm{ml}$ of venous blood obtained from newborns were drawn into bottles with $10 \mathrm{ml}$ of Tripticase Soy Broth supplemented (Roche) and incubated to $37^{\circ} \mathrm{C}$. After $24 \mathrm{~h}$, the blood cultures were inoculated into Thioglycolate Broth (DIFCO) and plated on Blood Agar and Eosin-Methylene Blue Agar (EMB, DIFCO). The Thioglycolate Broth, and plates of Blood Agar and EMB Agar were incubated at $37^{\circ} \mathrm{C}$ during a period ranged from 18 to $24 \mathrm{~h}$. When the blood cultures were negative after incubation of $24 \mathrm{~h}$, the inoculation into broth and plates above cited were repeated during a week. Identification of $P$. aeruginosa strains were performed using the Crystal System of identification for fermenters and non-fermenters (BBL/Becton-Dickinson).

P. aeruginosa sepsis were defined by a single positive blood culture associated with appropriate clinical manifestations (one of the following clinical signs or symptoms: fever $>38^{\circ} \mathrm{C}$, hypothermia $<36.5^{\circ}$, apnoea, bradycardia or tachycardia) according to CDC definitions (Garner et al. 1988)

The antimicrobial susceptibility test was carried out through of disk diffusion method according to National Committee for Clinical Laboratory Standards, NCCLS (1997) recommendations. Quality control was carried out using standard strains of Escherichia coli (ATCC 25922), P. aeruginosa (ATCC 27953) and Staphylococcus aureus (ATCC 25923). The following concentrations of antimicrobials drugs (CECON) were used: cephalothin $(30 \mu \mathrm{g})$, cefoxitin $(30 \mu \mathrm{g})$, ceftriaxone $(30 \mu \mathrm{g})$, cefuroxime $(30 \mu \mathrm{g})$, carbenicillin $(100 \mu \mathrm{g})$, cefepime $(30 \mu \mathrm{g})$, ceftazidime $(30 \mu \mathrm{g})$, piperacillin/tazobactam $(100 / 10 \mu \mathrm{g})$, ticarcillin/ clavulanic acid $(75 / 10 \mu \mathrm{g})$, imipenem $(10 \mu \mathrm{g})$, gentamicin $(10 \mu \mathrm{g})$, amikacin $(30 \mu \mathrm{g})$, ciprofloxacin $(5 \mu \mathrm{g})$, trimetho$\mathrm{prim} / \mathrm{sulfamethoxazole}(1.25 / 23.75 \mu \mathrm{g})$, chloramphenicol $(30 \mu \mathrm{g})$, tetracycline $(30 \mu \mathrm{g})$.

Serotyping method - An 18h Nutrient Agar culture (at $37^{\circ} \mathrm{C}$ ) of each strain was used as antigen. One $\mathrm{ml}$ of sterile saline solution $(0.85 \% \mathrm{NaCl})$ was drawn into tubes containing the Nutrient Agar cultures and mixed to produce a suspension containing the bacterial growth. The O-group was identified by slide agglutination with $P$. aeruginosa Antisera Kit (Denka Seiken Co. Ltd., Tokyo, Japan), according to Homma (1982) and Liu et al. (1983).

PFGE method - The strains were submitted to chromosomal DNA extraction and processing according to the procedures previously described by Sader et al. (1994). Nucleic acids present in agarose plugs were digested with $10 \mathrm{U}$ of SpeI restriction enzyme at $37^{\circ} \mathrm{C}$ during $20 \mathrm{~h}$, and the electrophoresis procedure was carried out in $1 \%$ agarose gels and running buffer containing $0.5 \mathrm{X}$ TBE with a CHEF DR III pulsed field electrophoresis systems (Bio-
Rad, California). Running conditions consisted of two ramps in sequence (ramp A consisted of an initial switch time of $0.5 \mathrm{sec}$, a final switch time of $25 \mathrm{sec}$, and a run time of $20 \mathrm{~h}$; ramp B consisted of an initial switch time of 30 $\mathrm{sec}$, a final switch time of $60 \mathrm{sec}$, and run time of $4 \mathrm{~h}$ ). The voltage was $6 \mathrm{~V} / \mathrm{cm}$ for both ramps, and the temperature was kept constant at $13^{\circ} \mathrm{C}$. Fragments were stained with ethidium bromide and photographed. All agarose gels were run twice to verify the reproducibility of the tests.

Band patterns were analyzed using GelCompar II (Applied Maths, Belgium), without using internal markers. The similarities between fingerprints were determined by construction of a similarity matrix using the Dice's coefficient with $1.5 \%$ position tolerance and optimization of $1 \%$, and a dendrogram generated using the UPGMA clustering algorithm. Definition of clonal structures of $P$. aeruginosa strains were made according to Tenover et al. (1995)

Epidemiological analysis of patients - For each patient, an epidemiological record with demographic and microbiologic data was done. These data were registered in an EXCEL 7.0 program (Microsoft) and analyzed later in Epi-Info program (version 6.04b; CDC, Atlanta, USA).

A control group of 70 newborns which did not acquire sepsis during the hospitalization in the NICU-HMAF from July 1997 to July 1998 was created. These control cases were randomly selected from a group of 720 patients that presented negative blood cultures, to evaluate the statistical significance of the demographics data. Most variables were compared using the odds ratios (ORs), 95\% confidence intervals $\left(\mathrm{CI}_{95}\right)$, chi-square $\left(\chi^{2}\right)$ and $\mathrm{P}$ values, except, mean age, mean of antimicrobial drugs used by patients before positive blood presentation and mean of antimicrobials days use before positive blood culture presentation, that were compared using the $t$-student test.

\section{RESULTS}

Table I shows the general demographic characteristics of the hospitalized newborns in NICU-HMAF, Rio de Janeiro city, Brazil, seized by $P$. aeruginosa sepsis and classified as HI cases. The mean age of these newborns was 12.5 days, and the major frequencies of $\mathrm{HI}$ were detected in male sex $(59.4 \%)$ and vaginal delivery $(81.2 \%)$. Twenty patients $(62.5 \%)$ received antimicrobial drugs before positive blood cultures presentation with mean use of 3.6 antimicrobials per patient and mean of 11.8 days use. A total of $87.5 \%$ of the patients were premature, $62.5 \%$ presented very low birth weight (VLBW), $40.6 \%$ had asphyxia and $31.2 \%$ presented Hyaline Membrane Disease (HMD).

The statistical results of the comparison between study and control groups (Table I) using OR, $\mathrm{CI}_{95}, \chi^{2}, \mathrm{P}$ values and $t$-student test, showed statistical significance in mean of antimicrobial drugs used by patients before positive blood culture presentation $(\mathrm{T}=3.00)$ and mean of antimicrobials days use before positive blood cultures presentation $(\mathrm{T}=2.22)$.

A total of 11 different antimicrobials drugs (Table II) were used by 20 patients $(62.5 \%)$ before positive blood cultures presentation, with major use frequency for ampicillin (18/20 patients-90\%), amikacin (17/20-85\%), cefotaxime $(9 / 20-45 \%)$ and oxacillin (9/20-45\%). 


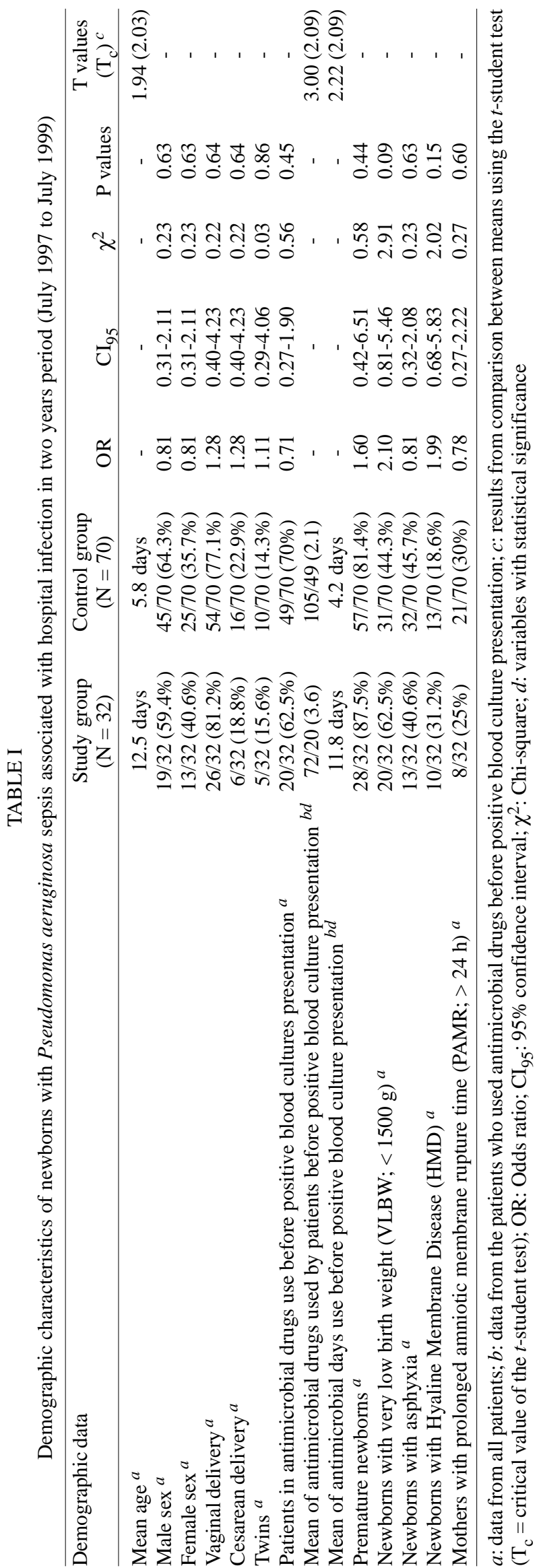

TABLE II

Antimicrobial drugs used by 20 newborns before positive blood culture presentation in two years period (July 1997 to July 1999)

\begin{tabular}{lcc}
\hline Antimicrobials & Frequency & $\%$ \\
\hline Ampicillin & 18 & 90 \\
Amikacin & 17 & 85 \\
Cefotaxime & 9 & 45 \\
Oxacillin & 9 & 45 \\
Imipenem & 7 & 35 \\
Ceftazidime & 4 & 20 \\
Vancomycin & 4 & 20 \\
Amphotericin & 1 & 5 \\
Ceftriaxone & 1 & 5 \\
Ciprofloxacin & 1 & 5 \\
Gentamicin & 1 & 5 \\
\hline Total & 72 & - \\
\hline
\end{tabular}

The antimicrobial resistance percentage of the isolated $P$. aeruginosa strains (Table III) demonstrated high antimicrobial resistance percentage ( 75 to $100 \%$ of resistance) to cephalotin, cefoxitin, ceftriaxone, cefuroxime, chloramphenicol, trimethoprim/sulfamethoxazole and tetracycline in two analyzed periods (July 1997 to July 1998 and August 1998 to July 1999); high antimicrobial resistance percentage to ceftazidime, cefepime, carbenicillin and ticarcillin/clavulanic acid during the first analyzed period and no resistant strain in the second; low antimicrobial resistance percentage ( 0 to $35 \%$ of resistance) to piperacillin/tazobactam in the first period and no resistant strain in second; low antimicrobial resistance percentage to gentamicin and amikacin (10.7\% each) during the first period and were detected increase in antimicrobial resistance percentage in second (50 and 25\%, respectively). In the first period there was no isolation of resistant strains to imipenem and ciprofloxacin, but in the second period an emergence of resistant strains was detected.

Fig. 1 shows seven distinct PFGE patterns (A-G) observed in the 32 P. aeruginosa strains, where the clone A was the most frequent (epidemic clone; $75 \%$ of the strains), while the patterns $\mathrm{C}$ and $\mathrm{E}$ presented two strains, and the patterns B, D, F and G only one strain, corresponding to $25 \%$ of the isolated strains in total.

The dendrogram generated by GelCompar II (Fig. 2), revealed that the seven clones presented low similarity percentage $(<80 \%)$ under stringency conditions above cited, indicating that these clones are not genetically related.

The serotyping results (Table IV) demonstrated four serotypes, with isolation predominance of the serotype $\mathrm{E}$ (29 strains; $90.7 \%$ ), followed by serotypes B, D and I (one strain in each; 3.1\%). The correspondence nomenclature of the serotypes B, D, E and I with the International Antigenic Typing Scheme (IATS) serotypes designation are respectively: O2/O5/O16, O9, O11 and O1 (Homma 1982, Liu et al. 1983).

The distribution of serotypes according to the chromosomal profiles (PFGE) and isolation periods is shown 
TABLE III

Antimicrobial resistance percentage detected in 32 Pseudomonas aeruginosa strains isolated in a two years period (July 1997 to July 1999)

\begin{tabular}{|c|c|c|c|c|c|}
\hline Antimicrobials & $\begin{array}{c}07 / 97 \text { to } 07 / 98^{a} \\
(\mathrm{~N} / \%)^{c}\end{array}$ & $\begin{array}{c}08 / 98 \text { to } 07 / 99^{b} \\
(\mathrm{~N} / \%)^{c}\end{array}$ & Antimicrobials & $\begin{array}{c}07 / 97 \text { to } 07 / 98^{a} \\
(\mathrm{~N} / \%)^{c}\end{array}$ & $\begin{array}{c}08 / 98 \text { to } 07 / 99^{b} \\
(\mathrm{~N} / \%)^{c}\end{array}$ \\
\hline Cephalothin & $28 / 100$ & $4 / 100$ & Ticarcillin/clavulanic acid & $22 / 78.6$ & $0 / 0$ \\
\hline Cefoxitin & $28 / 100$ & $4 / 100$ & Imipenem & $0 / 0$ & $2 / 50$ \\
\hline Ceftriaxone & $27 / 96.4$ & $4 / 100$ & Gentamicin & $3 / 10.7$ & $2 / 50$ \\
\hline Cefuroxime & $28 / 100$ & $4 / 100$ & Amikacin & $3 / 10.7$ & $1 / 25$ \\
\hline Ceftazidime & $26 / 92.9$ & $0 / 0$ & Ciprofloxacin & $0 / 0$ & $1 / 25$ \\
\hline Cefepime & $22 / 78.6$ & $0 / 0$ & Chloramphenicol & $26 / 92.9$ & $4 / 100$ \\
\hline Carbenicillin & $24 / 85.7$ & $0 / 0$ & Trimethoprim/sulfamethoxazole & $25 / 89.3$ & $4 / 100$ \\
\hline Piperacillin/tazobactam & $9 / 32.1$ & $0 / 0$ & Tetracycline & $21 / 75$ & $4 / 100$ \\
\hline
\end{tabular}

$\bar{a}: 28$ strains were isolated during this period; $b: 4$ strains were isolated during this period; $c$ : number/percentage of isolated resistant strains in period

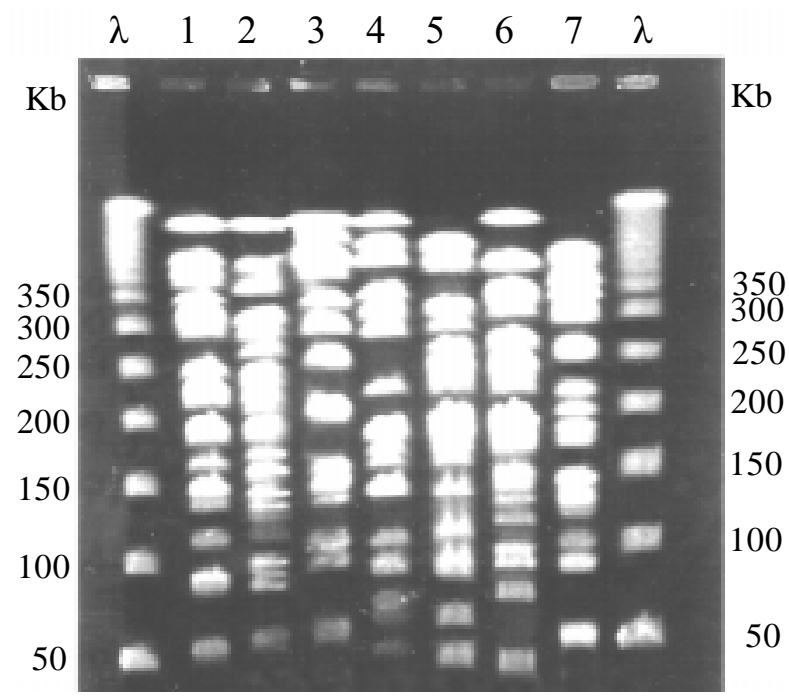

Fig. 1: $\lambda$ - pulsed field gel electrophoresis marker (lambda ladder 50 to $1000 \mathrm{~Kb}$ ); Lanes 1-7: pulsed field gel electrophoresis patterns detected in 32 strains of Pseudomonas aeruginosa isolated from blood culture of neonatal intensive care unit newborns. 1: A (24 strains); 2: B (1 strain); 3: C (1 strain); 4: F (2 strains); 5: G (1 strain); 6: E (2 strains); 7: D (1 strain)

in Table IV. Strains with serotype O11 and chromosomal profile A (23 strains; $71.9 \%$ ) were the more prevalent and have been isolated from July 1997 to June 1998. The others serotypes and chromosomal profiles were detected in small number distributed along the study period.

The distribution of chromosomal profiles and serotypes according to antimicrobial resistance profile (ARP) (Table V) showed 16 ARP including resistance ranged from 6 to 13 antimicrobials. Resistance profile $12(28.1 \%)$ was the more frequent, followed by the profiles 13 and 15 , with $12.6 \%$ of the isolates in each. Strains belonged to serotype O11 (90.7\% of the strains) were distributed in 14 different ARPs (all, except ARPs 1 and 5), and demonstrated antimicrobial resistance varying from 6 to 13 antibiotics. The strains with PFGE pattern A (75\% of the strains) were distributed in 10 different ARPs $(1,4,6-9,12-$
15), with antimicrobial resistance varying from 6 to 12 antimicrobials.

\section{DISCUSSION}

$P$. aeruginosa is the fourth most frequent pathogen isolated from nosocomial sepsis cases in the NICU-HMAF, accounting for $12.5 \%$ (32/255) of the infection cases, during the study period (data not shown). From the 32 sepsis cases detected in two years, $28(87.5 \%)$ were detected in the first year of the study period. Sader (2000) related similar results notifying that $P$. aeruginosa was the third most frequent pathogen isolated from Gram-negative sepsis cases in a multi-centric study performed in Brazil, accounting $14.6 \%(125 / 855)$ of the cases. In another study on bloodstream infection cases in Latin America (Sader et al. 1999), 736 isolates were analyzed and P. aeruginosa was the fourth more frequent pathogen isolated in these cases, accounting $6.9 \%(51 / 736)$ of the isolates.

The newborns seized by $P$. aeruginosa sepsis in the HMAF, when compared with the control group presented as risk conditions for $\mathrm{HI}$ acquisition, a more elevated mean of antimicrobials drugs used before positive blood culture presentation (3.6 vs 2.1) and a major time use (11.8 days vs 4.2 days) of these drugs, during the study period. These observations agree with other authors who described the extensive antimicrobial drugs use as predisposing risk factor to $\mathrm{HI}$ acquisition in NICUs, because these extensive use can select multi-drug resistant microorganisms (Jones et al. 1997, Leroyer et al. 1997, Cordero et al. 1999, Brodie et al. 2000).

Due the clinical picture suggestive of sepsis showed by infected newborns, a large number of patients $(62.5 \%)$ received antimicrobials drugs before positive blood culture presentation with means use of 3.6 antimicrobials per patient and 11.8 days. In these cases, co-administration of ampicillin plus amikacin was the first empirical therapeutic scheme adopted in our institution, followed by oxacillin plus cefotaxime (second scheme). These results associated with high levels of antimicrobial resistance detected in the isolated strains, reinforce the idea that the empirical treatment adopted in hospital routine induced selective pressure of multi-drug resistant strains. Several authors described that prolonged use of antimicrobials 


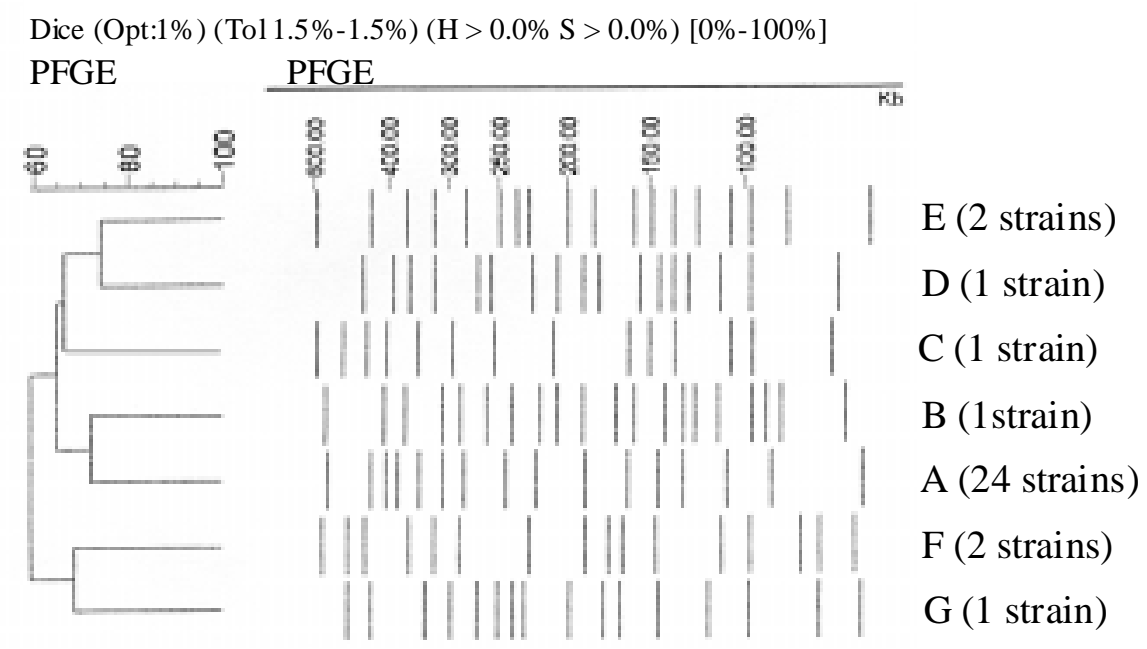

Fig. 2: dendrogram of the seven (A-G) pulsed field gel electrophoresis patterns (PFGE) detected in 32 Pseudomonas aeruginosa strains isolated from blood culture of neonatal intensive care unit newborns.

before and during NICU stay can lead to selection of multiresistant $P$. aeruginosa strains with different resistance forms, resulting in treatment failure, due selective pressure promoted by indiscriminated use of antimicrobials, principally broad-spectrum antimicrobials (Kettner et al. 1995, Bert \& Lambert-Zechovsky 1996, Cailleaux et al. 1997, Cordero et al. 1999).

In relation to antimicrobial resistance, cephalothin, cefoxitin, ceftriaxone, cefuroxime, chloramphenicol, tetracycline and trimethoprim/sulfamethoxazole, should not be considered effective agents for $P$. aeruginosa sepsis treatment in our hospital unit, due the high resistance rates detected during the study period. Others authors detected high resistance levels to $\beta$-lactams and trimethoprim/ sulfamethoxazole in hospital units (Sader et al. 1993, Kettner et al. 1995).

We detected high resistance percentage for ceftazidime, cefepime, carbenicillin and ticarcillin/ clavulanic acid during the first year of analysis and only sensible strains during the second year. These results indicate that resistance to these drugs were most associated with the epidemic strains isolated during the first year, in second year were no isolated these strains, and consequently these drugs can be newly considered effective in treatment of $P$. aeruginosa sepsis at HMAF routine. Others studies recommend the use of ceftazidime, carbenicillin and cefepime for $P$. aeruginosa sepsis treatment (Ismaeel 1993, Sader 2000). For ticarcillin/clavulanic acid, some studies related that this antimicrobial was little active against $P$. aeruginosa strains (Ismaeel 1993, Tassios et al. 1998).

In a multi-centric study, performed by Sader (2000) in Brazil, were detected that imipenem is the most active compound against $P$. aeruginosa infections followed by ciprofloxacin. These drugs are considered the most effective agents for treatment of $P$. aeruginosa sepsis at HMAF, but some resistant strains were isolated in the second period of analysis (August 1998 to July 1999). Several authors consider imipenem and ciprofloxacin as potent agents in treatment of infections caused by multi-resistant $P$. aeruginos $a$ and alerted the emergence of mutants for these drugs in the last years, due the indiscriminate use of these antimicrobials (Jones et al. 1997, Sader 2000, Tsakris et al. 2000).

Gentamicin and amikacin are considered effective antimicrobial agents and are largely used in the HMAF routine, but were detected a low number of resistant strains at HMAF during two years of study. This fact reflect the importance of to control the use of these antimicrobials in the hospital unit, for preventing the emergence of aminoglycosides-resistant strains. In addition, MüllerPremru and Gubina (1999) recommend the start of the restriction of antimicrobials use, when aminoglycosidesresistant strains are detected. Gentamicin and amikacin are considered by some authors as a suitable aminoglycoside antibiotics against drug resistant $P$. aeruginosa (Kettner et al. 1995, Jones et al. 1997).

We detected low number of resistant strains to piperacillin/tazobactam in the first year of study and none during the second year, suggesting that these drugs can be considered as effective agents in $P$. aeruginosa sepsis therapy. Sader (2000) related that piperacillin/tazobactam is the third most active compound in a multi-centric study of antimicrobial resistance in Brazil. The use of piperacillin, as well as others $\beta$-lactams must be monitored, because these antibiotics induce selective pressure of $\beta$-lactamase producers strains, resulting in resistance development in the hospital unit (Cailleaux et al. 1997).

Different resistance mechanisms have been observed in $P$. aeruginosa, such as reduced permeability of antimicrobials through outer membrane, antimicrobial efflux mechanisms, changes in the lipopolysaccharide, modification of DNA gyrase protein and inactivation or modification of the antimicrobial structure through enzymes production (Bert \& Lambert-Zechovsky 1996, Cailleaux et al. 1997, Jones et al. 1997, Esparragón et al. 1999, Tsakris et 


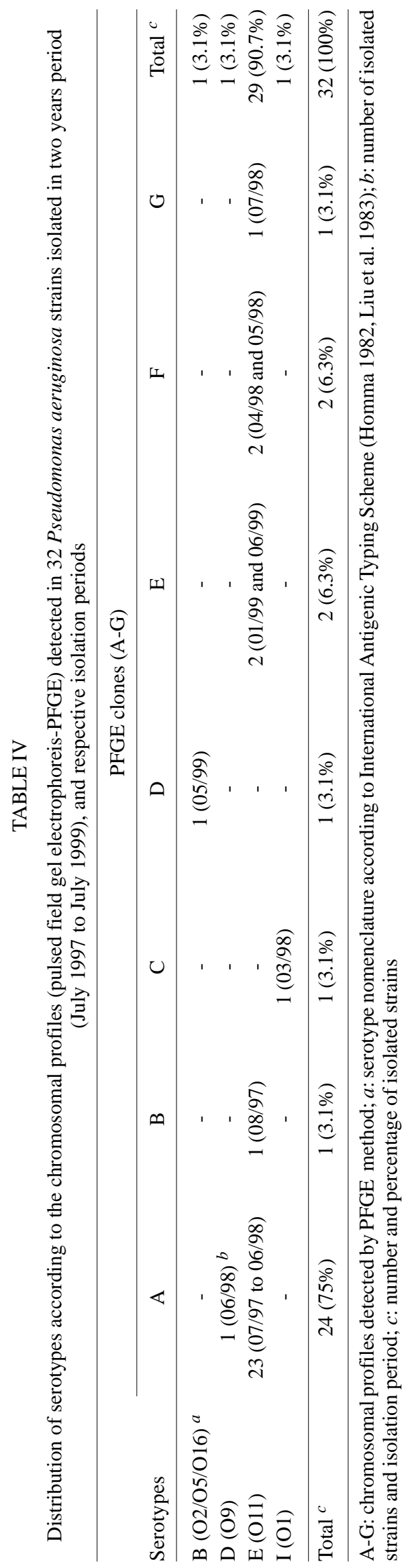

al. 2000). The 16 different ARPs observed in the strains isolated in this study presented resistance to antimicrobials include at least two different families of drugs with different action mechanisms, characterizing the analyzed strains as multi-drug resistant.

Several authors related that $P$. aeruginosa serotype $\mathrm{O} 11$ has been recognized as an important hospital problem in recent years, principally in epidemic situations, because this microorganism present multi-drug resistance with different resistance phenotypes (Pitt 1988, Kettner et al. 1995, Bert \& Lambert-Zechovsky 1996, Kinoshita et al. 1997, Tassios et al. 1998, Esparragón et al. 1999, MüllerPremru \& Gubina 1999). In this study, strains with serotype 011 showed 14 different ARPs with resistance varying from 6 to 13 antimicrobial drugs. This fact reflect the importance of controlling the emergence of strains with this serotype in NICUs.

The serotype O11 was the most prevalent (29/32$90.7 \%$ ), and in major number associated with PFGE pattern A $(23 / 29-79.3 \%)$ but was also associated with the PFGE patterns B, E, F and G (20.7\% in total). These associations demonstrated poor discriminatory power of the serotyping technique for epidemiologic studies application. The results showed by earlier studies, recommends serotyping (simple, cheap, fast, and present good reproducibility) as an initial screening procedure in epidemiological studies of $P$. aeruginosa (Renders et al. 1996, Bergmans et al. 1997). On the other hands, PFGE analysis included one strain serotype O9 into the PFGE profile of the epidemic strains (PFGE pattern A). A similar observation was described by Grundmann et al. (1995) in an study of 77 P. aeruginosa from unrelated sources in London, demonstrating genetically closely related strains from unrelated sources and different serotypes.

The strains with PFGE pattern A were considered as an epidemic clone restrict to the first year of analysis, this is probably due to elimination of these strains from hospital environment after reinforcement of HI prevention measures (measures of contact isolation such as: suitable handwashing, gloving and gown use for management of the newborns) and control (treatment of the infected newborns) in our hospital unit, initiated in May 1998 with the objective of to control a MRSA (methicillin-resistant $S$. aureus) outbreak, as previously described (Loureiro et al. 2000). After reinforcement of the prevention and control measures, no new outbreaks caused by MRSA and $P$. aeruginosa was observed in our hospital unit.

The epidemic strains (PFGE pattern A) showed 10 different ARPs, with resistance varying from 6 to 12 antimicrobials, that can be associated with selective pressure in hospital environment, principally in NICUs, resulting in resistance development. In addition, Harris et al. (1999) demonstrated that serial isolates with different antimicrobial profiles from individual patients represented the same strain, after typing though PFGE methodology, indicating that resistance to each class of antipseudomonal agents emerged sequentially after antibiotic exposure. Therefore, the multi-drug antibiogram is important to verify the emergence of resistance in the NICU, but resistance profile analysis is not a suitable epidemiological marker for detection of $P$. aeruginosa outbreaks, because changes in 
TABLE V

Distribution of chromosomal profiles (pulsed field gel electrophoresis) and serotypes in relation to antimicrobial resistance profiles (ARP) detected in 32 Pseudomonas aeruginosa strains isolated in two years period (July 1997 to July 1999)

\begin{tabular}{|c|c|c|c|c|}
\hline $\begin{array}{l}\text { ARP } \\
\text { no. }\end{array}$ & ARP & $\begin{array}{c}\text { No. of } \\
\text { strains (\%) }\end{array}$ & Serotypes ${ }^{a}$ & Clones $(\mathrm{N})$ \\
\hline 1 & CFL/CFO/CRO/CRX/CLO/TET & $1(3.1)$ & $\mathrm{D}(\mathrm{O} 9)$ & A (1) \\
\hline 2 & CFL/CFO/CRX/CLO/SXT/TET & $1(3.1)$ & $\mathrm{E}(\mathrm{O} 11)$ & $\mathrm{F}(1)$ \\
\hline 3 & CFL/CFO/CRO/CRX/CLO/SXT/TET & $2(6.3)$ & $\begin{array}{c}\mathrm{E}(\mathrm{O} 11) \text { and } \\
\mathrm{B}(\mathrm{O} 2 / \mathrm{O} 5 / \mathrm{O} 16)\end{array}$ & $\mathrm{E}(1), \mathrm{D}(1)$ \\
\hline 4 & CFL/CFO/CRO/CRX/CAZ/CAR/GEN/AMI & $1(3.1)$ & $\mathrm{E}(\mathrm{O} 11)$ & A (1) \\
\hline 5 & CFL/CFO/CRO/CRX/CAZ/CLO/SXT/TET & $1(3.1)$ & $\mathrm{I}(\mathrm{O} 1)$ & $\mathrm{C}(1)$ \\
\hline 6 & CFL/CFO/CRO/CRX/CAZ/CAR/CLO/SXT/TET & $1(3.1)$ & $\mathrm{E}(\mathrm{O} 11)$ & A (1) \\
\hline 7 & CFL/CFO/CRO/CRX/CAZ/CPM/CAR/TIC/CLO/SXT & $2(6.3)$ & $\mathrm{E}(\mathrm{O} 11)$ & A (2) \\
\hline 8 & CFL/CFO/CRO/CRX/CAZ/CPM/CAR/TIC/CLO/TET & $1(3.1)$ & $\mathrm{E}(\mathrm{O} 11)$ & $\mathrm{A}(1)$ \\
\hline 9 & CFL/CFO/CRO/CRX/CAZ/GEN/AMI/CLO/SXT/TET & $1(3.1)$ & $\mathrm{E}(\mathrm{O} 11)$ & A (1) \\
\hline 10 & CFL/CFO/CRO/CRX/IMI/GEN/CIP/CLO/SXT/TET & $1(3.1)$ & $\mathrm{E}(\mathrm{O} 11)$ & $\mathrm{G}(1)$ \\
\hline 11 & CFL/CFO/CRO/CRX/IMI/GEN/AMI/CLO/SXT/TET & $1(3.1)$ & $\mathrm{E}(\mathrm{O} 11)$ & $\mathrm{E}(1)$ \\
\hline 12 & CFL/CFO/CRO/CRX/CAZ/CPM/CAR/TIC/CLO/SXT/TET & $9(28.1)$ & $\mathrm{E}(\mathrm{O} 11)$ & A (9) \\
\hline 13 & CFL/CFO/CRO/CRX/CAZ/CPM/CAR/PPT/TIC/CLO/SXT & $4(12.6)$ & $\mathrm{E}(\mathrm{O} 11)$ & $\mathrm{A}(3), \mathrm{F}(1)$ \\
\hline 14 & CFL/CFO/CRO/CRX/CAZ/CPM/CAR/PPT/TIC/SXT/TET & $1(3.1)$ & $\mathrm{E}(\mathrm{O} 11)$ & A (1) \\
\hline 15 & CFL/CFO/CRO/CRX/CAZ/CPM/CAR/PPT/TIC/CLO/SXT/TET & $4(12.6)$ & $\mathrm{E}(\mathrm{O} 11)$ & A (4) \\
\hline 16 & CFL/CFO/CRO/CRX/CAZ/CPM/CAR/TIC/GEN/AMI/CLO/SXT/TET & $1(3.1)$ & $\mathrm{E}(\mathrm{O} 11)$ & $\mathrm{B}(1)$ \\
\hline
\end{tabular}

$a$ : serotype nomenclature according to International Antigenic Typing Scheme (Homma 1982, Liu et al. 1983); A-G: detected clones among the isolated strains by PFGE method. CFL: cephalothin; CFO: cefoxitin; CRO: ceftriaxone; CRX: cefuroxime; CAZ: ceftazidime; CPM: cefepime; CAR: carbenicillin; PPT: piperacillin/tazobactam; TIC: ticarcillin/clavulanic acid; IMI: imipenem; GEN: gentamicin; AMI: amikacin; CIP: ciprofloxacin; CLO: chloramphenicol; SXT: trimethoprim/sulfamethoxazole; TET: tetracycline

the antibiogram profile can occur, particularly during a long outbreak (Sader et al. 1993, Müller-Premru \& Gubina 1999).

This study has epidemiological implication, because few studies exist on occurrence of sepsis cases caused by $P$. aeruginosa strains serotype $\mathrm{O} 11$ in NICU patients from Brazil and Latin America. Our observations emphasize the need for appropriate microbiological monitoring of $P$. aeruginosa strains implicated in hospital infections using both traditional and molecular methods, as well as, emergence monitoring of resistant strains to broad-spectrum antimicrobials in hospital environment, and the need to reinforce educational measures for prevention of nosocomial transmission of multi-drug resistant microorganisms.

\section{REFERENCES}

Bergmans D, Bonten M, Tiel FV, Gaillard C, London N, Geest SV, Leeuw P, Stobberingh 1997. Value of phenotyping methods as an initial screening of Pseudomonas aeruginosa in epidemiological studies. Infection 25: 350-354.

Bert F, Lambert-Zechovsky N 1996. Comparative distribution of resistance patterns and serotypes in Pseudomonas aeruginosa isolates from intensive care units and others wards. J Antimicrob Chemother 37: 809-813.

Brodie SB, Sands KE, Gray JE, Parker RA, Goldmann DA, Davis RB, Richardson DK 2000. Occurrence of nosocomial bloodstream infections in six neonatal intensive care units. Pediatr Infect Dis J 19: 56-65.

Cailleaux V, Mulin B, Capellier G, Julliot MC, Thouverez M, Talon D 1997. Epidemiological study of variations in $\beta$ lactam antibiotic susceptibility of Pseudomonas aeruginosa in two intensive care units. J Hosp Infect 37: 217-224.
Cordero L, Sananes M, Ayers LW 1999. Bloodstream infections in a neonatal intensive-care unit: 12 years'experience with an antibiotic control program. Infect Control Hosp Epidemiol 20: 242-246.

Esparragón FR, Martín MG, Lama ZG, Sabatelli FJ, Junco MTT 1999. Aminoglycoside resistance mechanisms in clinical isolates of Pseudomonas aeruginosa from the Canary Islands. Zent bl Bakteriol 289: 817-826.

Garner JS, Jarvis WR, Emori TG, Horan TC, Hughers JM 1988. CDC definitions for nosocomial infections. Am J Infect Control 16: 128-140.

Grundmann H, Schneider C, Hartung D, Daschner FD, Pitt TL 1995. Discriminatory power of three DNA-based typing techniques for Pseudomonas aeruginosa. J Clin Microbiol 33: 528-534.

Harris A, Torres-Vieira C, Venkataraman L, DeGirolami P, Samore M, Carmeli Y 1999. Epidemiology and clinical outcomes of patients with multiresistant Pseudomonas aeruginosa. Clin Infect Dis 28: 1128-1133.

Homma JY 1982. Designation of the thirteen O-group antigens of Pseudomonas aeruginosa; an amendment for the tentative proposal in 1976. Jpn J Exp Med 52: 317-320.

Ismaeel NA 1993. Colonization of intensive care unit patients by Pseudomonas aeruginosa. J Hosp Infect 25: 279-286.

Jones RN, Pfaller MA, Marshall SA, Hollis RJ, Wilke WW 1997. Antimicrobial activity of 12 broad-spectrum agents tested against 270 nosocomial blood stream infection isolates caused by non-enteric Gram-negative bacilli: occurrence of resistance, molecular epidemiology, and screening for metallo-enzymes. Diagn Microbiol Infect Dis 29: 187192.

Kettner M, Milosovic P, Hletková M, Kallová J 1995. Incidence and mechanisms of aminoglycoside resistance in Pseudomonas aeruginosa serotype $\mathrm{O} 11$ isolates. Infection 
23: 380-383.

Kinoshita M, Sawabe E, Okamura N 1997. Concept of segmentation in nosocomial epidemiology: Epidemiological relation among antimicrobial-resistant isolates of Pseudomonas aeruginosa. J Infect 35: 269-276.

Leroyer A, Bedu A, Lombrail P, Desplanques L, Diakite B, Bingen E, Aujard Y, Brodin M 1997. Prolongation of hospital stay and extra costs due to hospital-acquired infection in a neonatal unit. $J$ Hosp Infect 35: 37-45.

Liu PV, Matsumoto H, Kusama H, Bergan T 1983. Survey of heat-stable, major somatic antigens of Pseudomonas aeruginosa. Int J Syst Bacteriol 33: 256-264.

Loureiro MM, Moraes BA, Quadra MRR, Pinheiro GS, Suffys PN, Asensi MD 2000. Molecular epidemiology of methicillin resistant Staphylococcus aureus isolated from newborns in a hospital in Rio de Janeiro, Brazil. Mem Inst Oswaldo Cruz 95: 776-782.

Moolenaar RL, Crutcher JM, Joaquin VHS, Sewell LV, Hutwagner LC, Carson LA, Robison DA, Smithee LMK, Jarvis WR 2000. A prolonged outbreak of Pseudomonas aeruginosa in a neonatal intensive care unit: Did staff fingernails play a role in disease transmission? Infect Control Hosp Epidemiol 21: 80-85.

Müller-Premru M, Gubina M 1999. Serotype, antimicrobial susceptibility and clone distribution of Pseudomonas aeruginosa in a university hospital. Zent bl Bakteriol 289: 857-867.

NCCLS-National Committee for Clinical Laboratory Standards 1997. Methods for dilution antimicrobial tests for bacteria that grow aerobically. Approved standard M7-A4. NCCLS, Wayne, PA.

Pitt TL 1988. Epidemiological typing of Pseudomonas aeruginosa. Eur J Clin Microbiol Infect Dis 7: 238-247.

Poh CL, Yeo CC, Tay L 1992. Genome fingerprinting by pulsed field gel electrophoresis and ribotyping to diferentiate Pseudomonas aeruginosa serotype 011 strains. Eur J Clin Microbiol Infect Dis 11: 817-822.

Renders N, Römling U, Verbrugh H, Belkum AV 1996. Comparative typing of Pseudomonas aeruginosa by random amplification of polymophic DNA or pulsed field gel electrophoresis of DNA macrorestriction fragments. J Clin Microbiol 34: 3190-3195.

Sader HS 2000. Antimicrobial resistance in Brazil: comparison of results from two multicenter studies. BJID 4: 91-99.

Sader HS, Pfaller MA, Jones RN, Doern GV, Gales AC, Winokur PL, Kugler KC, the SENTRY Latin America Study Group 1999. Bacterial pathogens isolated from patients with bloodstream infections in Latin America, 1997: Frequency of occurrence and antimicrobial susceptibility patterns from SENTRY antimicrobial surveillance program. BJID 3: 97-110.

Sader HS, Pignatari AC, Hollis R, Jones RN 1994. Evaluation of interhospitalar spread of methicillin-resistant Staphylococcus aureus in São Paulo, Brazil, using pulsed field gel electrophoresis of chromossomal DNA. Infect Control Hosp Epidemiol 15: 320-323.

Sader HS, Pignatari AC, Leme IL, Burattini MN, Tancresi R, Hollis RJ, Jones RN 1993. Epidemiologic typing of multiply drug-resistant Pseudomonas aeruginosa isolated from an outbreak in an intensive care unit. Diag Microbiol Infect Dis 17: 13-18.

Severino P, Darini AL, Magalhães VD 1999. The discriminatory power of ribo-PCR compared to conventional ribotyping for epidemiological purposes. APMIS 107: 10791084.

Tassios PT, Gennimata V, Maniatis AN, Fock C, Legakis NJ, The Greek Pseudomonas aeruginosa study group 1998. Emergence of multi-drug resistance in ubiquitous and dominant Pseudomonas aeruginosa serogroup O:11. J Clin Microbiol 36: 897-901.

Tenover FC, Airbeit RD, Goering RV, Mickelsen PA, Murray BE, Persing DH, Swaminathan B 1995. Interpreting chromosomal DNA restriction patterns produced by pulsedfield gel electrophoresis: Criteria for bacterial Strain typing. J Clin Microbiol 33: 2233-2239.

Tsakris A, Pournaras S, Woodford N, Palepou MFI, Babini GS, Douboyas J, Livermore DM 2000. Outbreak of infections caused by Pseudomonas aeruginosa producing VIM-1 carbapenemase in Greece. J Clin Microbiol 38: 1290-1292. 